\title{
Erratum to: Improving near-infrared quantum-dot light-emitting diodes to mimic synaptic plasticity (vol 62, issue 10, page 1470, 2019)
}

Shuangyi Zhao ${ }^{1}$, Yue Wang ${ }^{1}$, Wen Huang ${ }^{1}$, Hao Jin ${ }^{1}$, Peiwen Huang ${ }^{1}$, Hu Wang ${ }^{1}$, Kun Wang ${ }^{1}$, Dongsheng $\mathrm{Li}^{1}$, Mingsheng $\mathrm{Xu}^{2}$, Deren Yang ${ }^{1}$ and Xiaodong $\mathrm{Pi}^{{ }^{*}}$

In the version of the article originally published in the volume 62, issue 10, 2019 of Sci. China Mater. (page 1470-1478, https://doi.org/10.1007/s40843-019-9437-9), wrong images of Figs 3 and 4 were used. The corrected version appears below: the units of the $x$-axes in Fig. $3 \mathrm{~h}$, the inset of Fig. $4 \mathrm{a}$, and Fig. $4 \mathrm{~b}$, d should be h, s, s, and s, respectively.
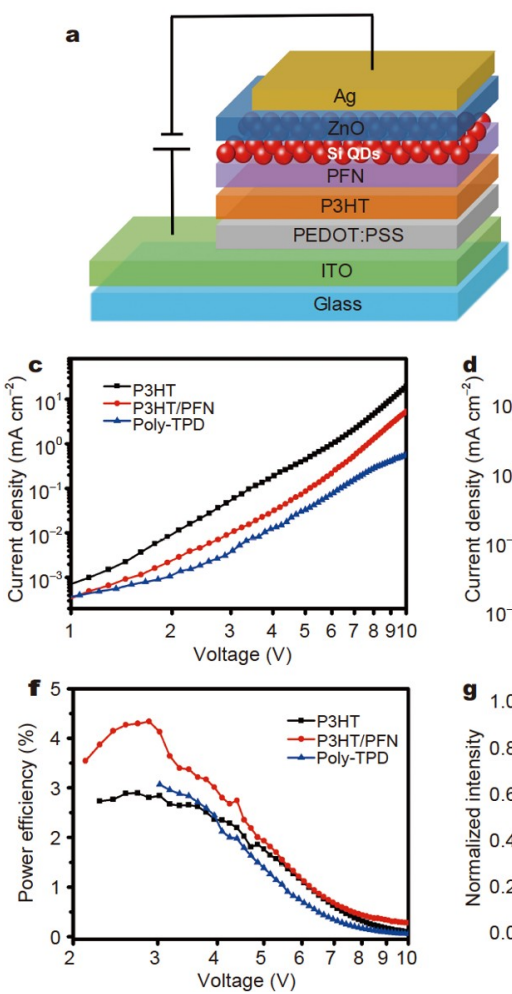
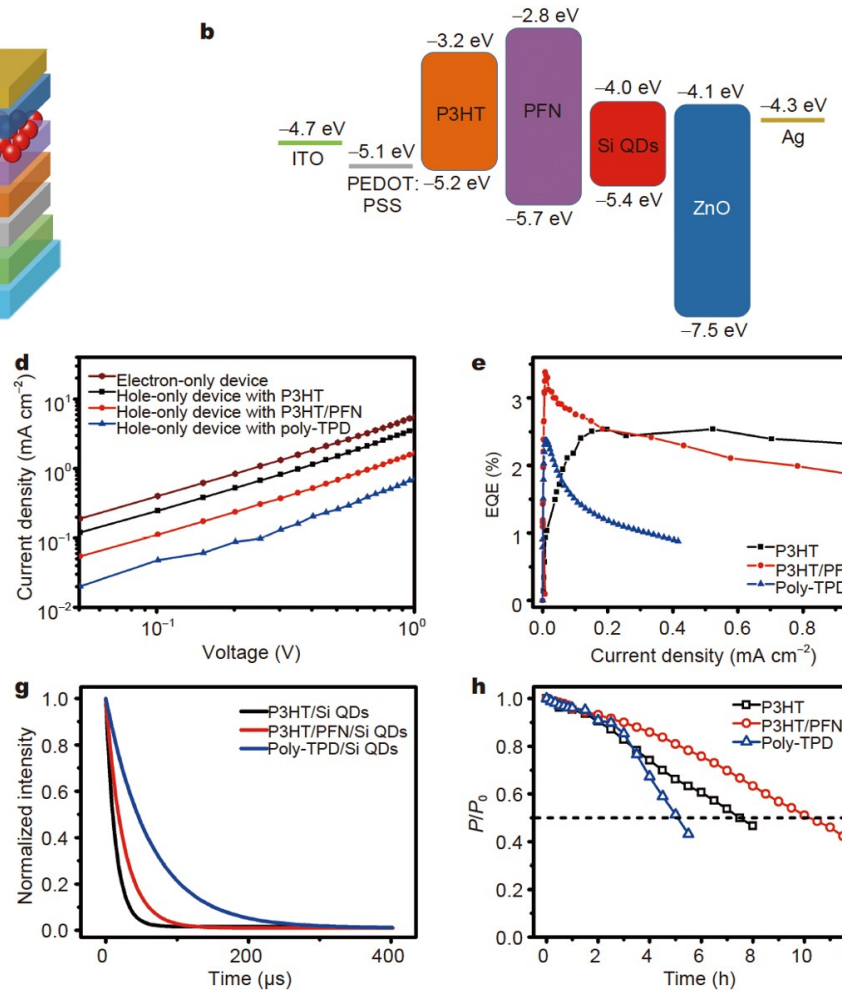
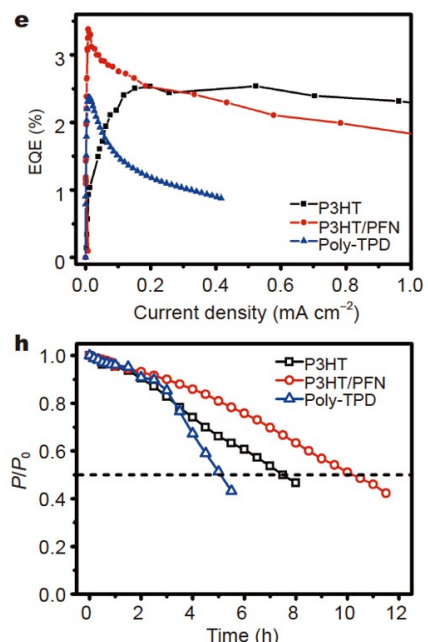

Figure 3 (a) Schematic diagram and (b) flat-band energy-level diagram of a NIR QLED based on Si QDs with a PFN interlayer. (c) $J-V$ curves for NIR QLEDs based on Si QDs with P3HT, P3HT/PFN and the traditional HTL of poly-TPD. (d) $J-V$ curves for an electron-only device and hole-only devices with P3HT, P3HT/PFN and poly-TPD. (e) EQE versus current density and (f) power efficiency versus voltage for NIR QLEDs based on Si QDs with P3HT, P3HT/PFN and poly-TPD. (g) PL decay curves of Si QDs with P3HT, P3HT/PFN and poly-TPD. (h) Stability of QLEDs with P3HT, $\mathrm{P} 3 \mathrm{HT} / \mathrm{PFN}$ and poly-TPD working at a driving voltage of $\sim 5 \mathrm{~V}$.

\footnotetext{
${ }^{1}$ State Key Laboratory of Silicon Materials \& School of Materials Science and Engineering, Zhejiang University, Hangzhou, Zhejiang 310027, China ${ }^{2}$ School of Information Science and Electronic Engineering, Zhejiang University, Hangzhou, Zhejiang 310027, China

* Corresponding author (email: xdpi@zju.edu.cn)
} 


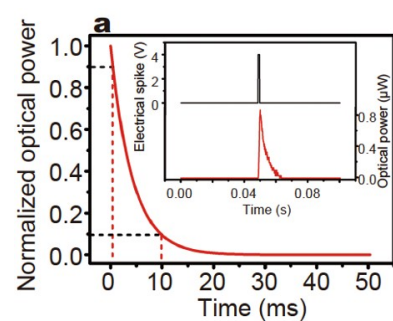

e

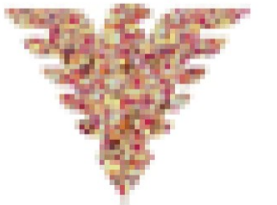

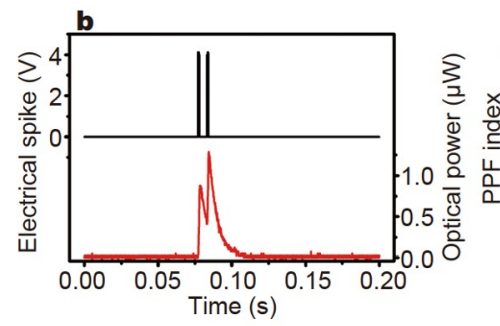

$\mathbf{f}$

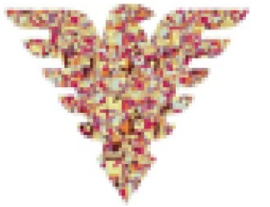

$$
\text { s }
$$

g

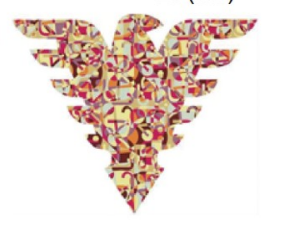

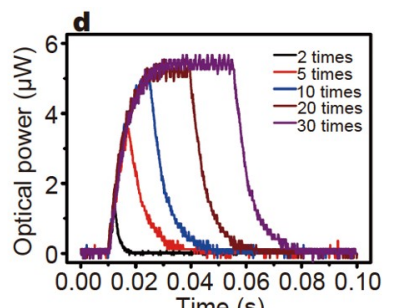

h

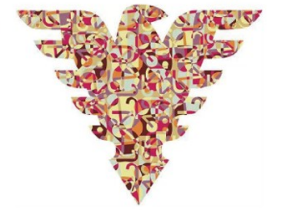

Figure 4 (a) Decay of the EL from a synaptic device stimulated by a $4 \mathrm{~V}$ electrical spike with a duration of $1 \mathrm{~ms}$. The electrical spike and the corresponding optical power of the EL are shown in the inset. (b) Optical power of a synaptic device induced by two successive electrical spikes. (c) Dependence of the PPF index on $\Delta t$ for $4 \mathrm{~V}$ electrical spikes. (d) Optical power of a synaptic device stimulated by from two to thirty times of $4 \mathrm{~V}$ electrical spikes. Image recognition depictions for a synaptic device stimulated by 2, 5, 10, and 20 successive electrical spikes are shown in (e-h). 